\title{
The Perspective of a Bank in Granting Credits: an Optimization Model
}

\author{
Roy Cerqueti* \\ University of Macerata \\ Department of Economic and Financial Institutions \\ Via Crescimbeni, 20 - Macerata, Italy. \\ Tel. +39 0733 2583246. Fax: +390733 2583205 . \\ Email: roy.cerqueti@unimc.it \\ Anna Grazia Quaranta \\ Alma Mater Studiorum - University of Bologna \\ Department of MatemateS, Faculty of Economics \\ Viale Filopanti, 5 - Bologna, Italy \\ Email: annagrazia.quaranta@unibo.it
}

March 14, 2011

\begin{abstract}
In this work, a model for legal financiers' strategies is presented, taking into account that the aim of a bank is to minimize the default probability of the funded company, constrained with reaching a certain profit level. To obtain our purpose, a stochastic dynamics optimization model is constructed and solved in closed form and a Monte Carlo simulation involving empirical data is also implemented. The financial strategies are thus obtained.
\end{abstract}

Keywords: Stochastic model; optimal control problem; firms' external financing.

${ }^{*}$ Corresponding author 


\section{Introduction}

In the breakthrough paper of Modigliani and Miller (1958), the authors used an arbitrage argument to prove the separability of corporate financing and investment decisions when perfect capital market assumptions hold. Modigliani and Miller's result can be summarized as stating the irrelevance of capital structure when evaluating a firm's value.

In actual fact, the life of a firm can be influenced by several events, the impact of which can drastically change the evolution of the dynamics associated with the firm's value. We focus in this paper on an issue relevant to company wealth, that is external financing.

Brennan and Schwartz (1978) is the starting point of quantitative studies in the search for optimal external financing strategy. They perform a numerical analysis to determine the optimal leverage when the wealth of the firms is subject to a diffusion process with constant volatility. Leland (1994) shows closed form solutions for debt values and equity values assuming infinite life for the debts. In Leland and Toft (1996), the (very restrictive) assumption of infinite life debt is removed.

More recently, Sethi and Taksar (2002) consider the problem of searching for the best financing mix of retained earnings and external equity in a stochastic framework, in order to maximize a firm's value. For their purpose, they formulate and solve explicitly a singular stochastic control problem. Sethi and Taksar (2002) is the stochastic extension of the deterministic model stated in Krouse and Lee (1973) and improved in Sethi (1978).

Caballero and Pindyck (1996) examine the sources of randomness in company investments and the effects of external financing on the incomes of an industrial system. The approach they take is dynamic optimization, with a dynamic programming perspective. The authors extend and complement Dixit (1989) and Leahy (1991): indeed, on one hand, they adopt the viewpoint of these papers and focus on the entry or exit decisions, while on the other hand, in contrast with the quoted papers, they emphasize the effects of different sources of uncertainty on company financing policies.

A different perspective can be found in Cifarelli et al. (2002). The authors develop a model for choosing the best payment flow which a legal or illegal financier has to demand from a firm in order to have a debt repaid. Cifarelli et al. (2002) extend Masciandaro et al. (1997), and analyze the ruin probability of the firm via differential equation theory. The firm's wealth evolves according to a stochastic differential equation. A similar approach is also 
found in Li et al. (1996) in a different setting, where the stochastic intensity of the debt restitution appears in the drift coefficient as an additive term.

In this work, the general framework of Cifarelli et al. (2002) is adopted, and a company and a financier operating in a dynamic stochastic environment are analyzed, from the financier's point of view. Definitively, we construct and develop an optimization problem to search for the best possible intensity of payments which a bank should obtain from a firm in order to have a debt repaid.

A suitable model for external financing should take the financier's target into account. In this respect, a bank aims at maximizing its profit, but tries to avoid the failure of the company. The competitive scopes are pursued by the bank as follows.

An attempt is made to avoid failure of the funded company by stating a maximum distance-to-default problem. In doing so, the bank refers to the evolution of company wealth dynamics, which is implicitly assumed to be known. In fact, the bank checks the financial situation of the company before granting credit.

With respect to the minimum profit threshold, this is protected by the introduction of intermediation fees and by credit spreads. The intermediation fees are charged by the bank directly on the annuities paid by the funded company, while the credit spread contracts concern the transfer of the risk from the bank (protection buyer) to a third actor (protection seller). Obviously, the bank also charges interest on the credit annuities. Therefore, a constraint on the intensity of payments grounded on interest and fees is needed.

In addition, the problem is studied up to the time in which the debt is completely repaid or the company fails. Since the date of any success or failure of the firm is not fixed a priori, our optimization problem has a stochastic time horizon, endogenously determined by the dynamics of the firm's wealth. Cifarelli et al. (2002) is the literature contribution closest to our paper, in that we deal with the same problem -external financing of firms after credit has been granted- from the same perspective -that of the financier. Our approach differs from Cifarelli et al. (2002), in that we construct and solve a stochastic control problem in a dynamic programming framework. Several monographs give a complete overview of stochastic control theory. To cite some excellent references, we remind the reader to Borkar (1989), Fleming and Soner (1993), Krylov (1980) and Yong and Zhou (1999). For a collection of optimization techniques applied to real life problems, refer also to Pardalos 
and Tsitsiringos (Eds., 2002).

The model of Cifarelli et al. (2002) is so general, that it may be of interest both to financial economists and mathematicians, as the authors stress in the concluding section. We totally agree with this view, and we would like to point out that our dynamic programming approach is the first to improve on Cifarelli et al. (2002) in two important respects. First of all, we obtain an easy, explicit form of the value function through the closed form solution of the dynamic programming differential equation, while Cifarelli et al. (2002) simply provide a complicated series expansion of the ruin probability of a funded company. Secondly, in contrast with the paper we have quoted, we are able to pinpoint the optimal strategies a bank should apply to have back a loan in closed form.

This paper also provides a contribution to the debate on credit analysis. Indeed, an appropriate calibration of the model on real data may allow it to be a suitable simple instrument for banks to evaluate the structural credit spread (we refer to the models of Merton (1974), Longstaff and Schwartz (1995), Duan (1994), Duan et al. (2004)) or, more generally, customers' credit risk (see Remark 1). In this respect, the maximum value of the distance-todefault function -the definitive aim of this research- may give insights into the financial stability of the funded company. Unquestionably, this is a critical and urgent subject to meriting further close attention in these times of crisis. We refer to Section 4 for discussion of this topic.

The remaining part of the paper is organized as follows. The next section is devoted to stating the optimization problem. Section 2 contains the solution of the problem via dynamic programming and the explicit expressions of the optimal strategies. Section 3 contains a validation of our theoretical results through a Monte Carlo simulation. In Section 4, we present a discussion of the relationship between the financial stability of the funded company and the solidity of the financing bank. The final section concludes.

\section{The optimization problem}

We consider a company whose wealth at time $t$, denoted by $X(t)$, is described by a controlled stochastic differential equation, as we shall see.

We also introduce a probability space with filtration $\left(\Omega, \mathcal{F},\left\{\mathcal{F}_{t}\right\}_{t \geq 0}, P\right)$, where 
the filtration $\mathcal{F}_{t}$ is assumed to be cadlag and is constructed as

$$
\mathcal{F}_{t}=\operatorname{sigma}(X(s), \quad 0 \leq s \leq t) \cup \mathcal{N}, \forall t \geq 0,
$$

with

$$
\mathcal{N}:=\{A \in \mathcal{F} \mid P(A)=0\} .
$$

The state equation describes the stochastic evolution of the dynamic associated to the wealth of the firm. It is given by the following controlled stochastic differential equation with initial data.

$$
\left\{\begin{array}{c}
\mathrm{d} X(t)=(\mu X(t)-\alpha(t)) \mathrm{d} t+\sigma X(t) \mathrm{d} W(t), \\
X(0)=X_{0}
\end{array}\right.
$$

where

- $\mu, \sigma \in \mathbf{R}$ are related, respectively, to the deterministic and stochastic evolution of the firm's wealth.

- $\alpha(\cdot)$ is a stochastic process $\mathcal{F}_{t}$-adapted $\left(\alpha_{t}\right.$ belongs to $\mathcal{F}_{t}$ ), and it represents the intensity of payment corresponded by the funded firm to the financier.

- $X_{0} \in[0, K]$ is the initial wealth of the firm. Formally, it should be an integrable random variable in $[0, K]$ with law $\pi_{0}$, that is measurable with respect to $\mathcal{F}_{0}$. Since it is reasonable that the initial situation of the funded company is known, we can assume that $X_{0}=x \in[0, K], x$ nonrandom.

- $W(\cdot)$ is a standard 1-dimensional brownian motion that is independent of $\pi_{0}$. It drives the stochastic term of the firm's wealth evolution.

Remark 1. The dynamics of the value of the firm in (1) was introduced in Merton (1974) in the context of pricing models for corporate liabilities using an option valuation approach; this model and its extensions -among them Duan (1994), Duan et al. (2004), Longstaff and Schwartz (1995), Anderson and Sundaresan (2000)- are typically referred to as structural credit spreads or risky bond models. In agreement with Modigliani-Miller Theorem, the term $\alpha(\cdot)$ in Merton (1974) is, if positive, the total dollar payout by the firm 
per unit time to its shareholders or liability-holders-i.e. dividends or interest payments, respectively- while, if negative, the net amount in dollars received by the firm from new financing. In our case, we deal with positive $\alpha(\cdot)$, and we refer specifically to interest payments. This interpretation of the process $\alpha(\cdot)$ in equation (1) should be taken into account to explain the meaning of the distance-to-default of the funded company and the related value function of the optimization problem (see also Barone et al., in press).

Remark 2. The bound values 0 and $K$ are absorbing barriers for the dynamic of the wealth of the firm, which evolves under the pressure of the payment of the debt. When the wealth of the firm reaches the value 0 , then we have the company's failure; if the firm's wealth reaches the value $K$, then the loan can be extinguished. The restitution threshold $K$ has to be interpreted as a level of firm's wealth where the funded company is able, but not obliged to repay the debt.

Remark 3. There exists a unique solution for the controlled equation (1) (we remind the reader, for example, to Øksendal 1995)).

By Remark 3, and fixed $x \in(0, K)$ and $\alpha \in \mathbf{R}^{+}$, we denote the unique solution of (1) as $X_{x}^{\alpha}(\cdot)$.

Let us denote with $\mathcal{T}$ the set of the stopping times in $[0,+\infty)$, i.e.

$$
\mathcal{T}:=\left\{\tau: \Omega \rightarrow[0,+\infty] \mid\{\tau \leq t\} \in \mathcal{F}_{t}, \forall t \geq 0\right\},
$$

and let us define the exit time $\tau_{(0, K)}$ of the dynamic from $(0, K)$ as

$$
\tau_{(0, K)}:=\inf \left\{t \geq 0 \mid X_{x}^{\alpha}(t) \notin(0, K)\right\},
$$

Since $\mathcal{F}_{t}$ is cadlag, then $\tau \in \mathcal{T}$.

The optimization problem reflects the financial targets of the bank, when financing a company. With this respect, two competitive scopes, as already preannounced in the Introduction, should be taken under consideration in defining the value function and the admissible region:

- the default of the funded company should be avoided. Indeed, if the company were to fail, financial distress would also be experienced by the bank, since there would be no real guarantees of repayment of even part of the loan; 
- a minimum profit threshold should be obtained by the bank.

Hence, the value function $V$ is

$$
V(x):=\min _{\alpha \in \mathcal{A}} P\left(X_{x}^{\alpha}\left(\tau_{(0, K)}\right)=0\right),
$$

where the admissible region of the problem is

$$
\begin{gathered}
\mathcal{A}:=\left\{\alpha:[0,+\infty) \times \Omega \rightarrow\left[\delta_{1}, \delta_{2}\right] \subset \mathbf{R}^{+}\right. \text {such that } \\
\left.K_{1}+\mathbb{E}\left[\int_{0}^{\tau_{(0, K)}} \alpha_{t} e^{-\delta t} \mathrm{~d} t\right] \geq K_{2}, \quad \alpha_{t} \in \mathcal{F}_{t}, \quad \forall t \geq 0\right\} .
\end{gathered}
$$

$e^{-\delta}$ is the uniperiodal discount factor, and it is assumed to be constant, in line with Cifarelli et al. (2002). It has to be intended as a market discount factor.

$\delta_{1}$ and $\delta_{2}$ are, respectively, the lower and the upper bounds for the intensity of payments. They can be obtained by multiplying the minimum and maximum possible loan interest rates to the initial amount of the debt ${ }^{1}$.

$V$ formalizes the problem of the maximization of the distance to company's default, that is a standard target in credit risk theory (see Remark 1).

$K_{1}$ and $K_{2}$ are nonnegative constants: $K_{2}$ is the amount of the original debt of the firm while $K_{1}$ is an initial amount that the funded company has to pay to the bank, and it concerns the bureaucracy expenses needed to establish the terms of the contract. It is worth noting that if an equity condition holds, then $K_{2}$ should be equal to the expected discounted value of the annuities $\alpha_{t}$, with the addition of $K_{1}$. More formally, fairness of the loan imposes that

$$
K_{1}+\mathbb{E}\left[\int_{0}^{\tau_{(0, K)}} \alpha_{t} e^{-\delta t} \mathrm{~d} t\right]=K_{2}
$$

It is well-known that such a fairness is sometimes violated, and the LHS of equation (6) may be greater than the RHS. Therefore, the condition in (5) assumed in our model is more realistic, in that it is more general.

The presence of the budget constraint in (5) implies a change in the normal

\footnotetext{
${ }^{1}$ The loan interest rates are constrained in a range, that is theoretically determined in each Country by the laws against usury (see Masciandaro, 2001). In the actual practice, the loan interest rates vary in a narrower range, according also to the ranking of the funded company. As we shall see, the available data suggest us to adopt the latter perspective in our simulation analysis.
} 
upper and lower bound for the intensity of payments, which the financier must arrange with the firm. We should replace $\delta_{1}$ and $\delta_{2}$ with $\delta_{1}^{B}$ and $\delta_{2}^{B}$. Typically, the only change appears on the lower bound $\delta_{1}$, since $\delta_{2}$ cannot be responsible for violating profit conditions. A simple example of what happens follows.

Proposition 4. Suppose that $\alpha_{t}=\alpha \in \mathbf{R}^{+}$, for each $t>0$. Moreover, assume

$$
\delta_{1} \geq \delta\left[1+K_{2}-K_{1}\right]
$$

Then it results

$$
K_{1}+\mathbb{E}\left[\int_{0}^{\tau_{(0, K)}} \alpha_{t} e^{-\delta t} \mathrm{~d} t\right] \geq K_{2}
$$

Proof. If $\alpha_{t}=\alpha$ is constant, then (8) can be written as

$$
K_{1}+\mathbf{E}\left[\frac{\alpha}{\delta}\left(1-e^{-\delta \tau_{(0, K)}}\right)\right] \geq K_{2},
$$

that is equivalent to

$$
K_{1}+\frac{\alpha}{\delta}-\mathbf{E}\left[e^{-\delta \tau_{(0, K)}}\right] \geq K_{2}
$$

and so

$$
\alpha \geq \delta\left[K_{2}-K_{1}+\mathbf{E}\left[e^{-\delta \tau_{(0, K)}}\right]\right] \geq K_{2}
$$

Let us observe now that, for each $\omega \in \Omega, e^{-\delta \tau_{(0, K)}(\omega)} \in(0,1)$. Then (7) implies (9), that is equivalent to (8) and the result is completely proved.

We omit hereafter the superscript $B$ in $\delta_{1}$ and $\delta_{2}$, but we stress that the upper and lower bound of the admissible intensity of payments are to be read as modified by the intervention of the profit constraint condition in (5).

\section{Solution of the problem and optimal strate- gies}

The strategy we have adopted to solve the optimization problem is the dynamic programming approach. In this context, the value function can be viewed as a classical solution of a second order differential equation, namely the Hamilton Jacobi Bellman (HJB) equation. The proof of the dynamic programming equation follows from the related maximum principle. For detailed discussion, we refer to Fleming and Soner, (1993). We formalize the dynamic programming equation in our framework, and omit the proof. 
Theorem 5 (HJB Equation). Suppose that $V \in C^{2}((0, K)) \cap C^{0}(\{0, K\})$. Define

$$
H_{a}(x):=(\mu x-a) V^{\prime}(x)+\frac{1}{2} \sigma^{2} x^{2} V^{\prime \prime}(x) .
$$

Then

$$
\sup _{a \in\left[\delta_{1}, \delta_{2}\right]} H_{a}(x)=0, \forall x \in(0, K),
$$

with the boundary conditions

$$
V(0)=0, \quad V(K)=H
$$

where $H$ is a positive constant relative to the income of the financier, when the company is able to repay completely the debt.

The optimal strategies of the dynamic stochastic optimization problem we are studying are implied by the existence and uniqueness of the classical solution for equation (10), with boundary conditions (11), as we shall see below. Theorem 5 states that equation (10)-(11) can be written under some regularity conditions for $V$. The problem of proving the regularity of the value function, that is only assumed in Theorem 5 , is overcame in our model, since we find a closed form solution of equation (10)-(11). The following result holds.

Theorem 6. 1. There exists $a^{*} \in\left[\delta_{1}, \delta_{2}\right]$ such that

$$
H_{a^{*}}(x)=\sup _{a \in\left[\delta_{1}, \delta_{2}\right]} H_{a}(x)
$$

for each $x \in[0, K]$.

2. There exists an unique solution of the equation (10)-(11).

3. The function

$$
V(x)=C \int_{0}^{x} \exp \left\{-\frac{2 a^{*}}{\sigma^{2} t}-\frac{2 \mu}{\sigma^{2}} \log t\right\} \mathrm{d} t,
$$

with

$$
C=H \cdot\left(\int_{0}^{K} \exp \left\{-\frac{2 a^{*}}{\sigma^{2} t}-\frac{2 \mu}{\sigma^{2}} \log t\right\} \mathrm{d} t\right)^{-1},
$$

is the solution of the Hamilton Jacobi Bellman Equation (10)-(11). 


\section{Proof.}

1. Fixed $x \in[0, K]$, then $H_{a}(x)$ is a continuous function of the variable $a$ in the compact set $\left[\delta_{1}, \delta_{2}\right]$. By Weierstrass' Theorem, we obtain the thesis.

2. The proof comes out from the standard existence and uniqueness theorem for systems of first order ordinary differential equations.

3. Let us consider $a^{*}$ the same as in the first point of this theorem. (10) can be written as

$$
\left\{\begin{array}{c}
\left(\mu x-a^{*}\right) \gamma(x)+\frac{1}{2} \sigma^{2} x^{2} \gamma^{\prime}(x)=0, \quad x \in(0, K), \\
\gamma(x)=V^{\prime}(x), \quad x \in(0, K) .
\end{array}\right.
$$

By separating the variables, the first order differential equation in (15) becomes

$$
\frac{\mathrm{d} \gamma}{\gamma}=\frac{2\left(a^{*}-\mu x\right)}{\sigma^{2} x^{2}} \mathrm{~d} x
$$

and then

$$
\log \gamma(x)=-\frac{2 a^{*}}{\sigma^{2} x}-\frac{2 \mu \log x}{\sigma^{2}}+C_{1}, \quad C_{1} \in \mathbf{R} .
$$

Therefore we obtain

$$
\gamma(x)=C_{2} x^{-\frac{2 \mu}{\sigma^{2}}} \mathrm{e}^{-\frac{2 a^{*}}{\sigma^{2} x}} \quad C_{2} \in \mathbf{R}^{+} .
$$

These computations provide

$$
V(x)=\int_{0}^{x} \gamma(t) \mathrm{d} t=C_{2} \int_{0}^{x} t^{-\frac{2 \mu}{\sigma^{2}}} \mathrm{e}^{-\frac{2 a^{*}}{\sigma^{2}}}+C_{3}, \quad C_{3} \in \mathbf{R} .
$$

By imposing the boundary conditions (11), we get the thesis.

Remark 7. A direct consequence of Theorem 6 is that $V$ is strictly increasing in $(0, K)$. For a discussion of this result, see Section 4. 
The optimal strategies of our stochastic control problem can be theoretically identified by proving a Verification Theorem. To achieve this aim, we start from the HJB equation (10)-(11). More precisely, we use the results in Theorems 5 and 6 . We enunciate the Verification Theorem in our setting and we omit the proof, since our result is already contained in a more general theory. We remind the reader to Fleming and Soner (1993).

Theorem 8 (Verification Theorem). Assume that $u \in C^{0}([0, K]) \cap C^{2}(\{0, K\})$ be a solution of (10)-(11).

Then it results

- (a) $u(x) \geq V(x), \quad \forall x \in[0, K]$.

- (b) Let us consider $\left(\alpha^{*}, x^{*}\right)$ an admissible couple at $x$ such that

$$
\alpha^{*} \in \operatorname{argmax}_{a}\left\{\left(\mu x^{*}(t)-a\right) V^{\prime}\left(x^{*}(t)\right)+\frac{1}{2} \sigma^{2}\left(x^{*}(t)\right)^{2} V^{\prime \prime}\left(x^{*}(t)\right)\right\} .
$$

Then $\left(\alpha^{*}, x^{*}(t)\right)$ is optimal at $x$ and it results $u(x)=V(x), \forall x \in[0, K]$

Theorem 8 contains the formalization of the optimal controls related to our stochastic control problem. In particular, condition (20) implies that the optimal controls $\alpha^{*}$ are bang-bang controls. Indeed, by (20) we have:

$$
\alpha^{*}(x)= \begin{cases}\delta_{2} & \text { for } x \mid V^{\prime}(x)<0 \\ \delta_{1} & \text { for } x \mid V^{\prime}(x)>0 \\ \text { arbitrary } & \text { for } x \mid V^{\prime}(x)=0\end{cases}
$$

Since $V$ is strictly increasing, as already noticed above, we have that

$$
\Gamma:=\left\{x \in[0, K] \mid V^{\prime}(x) \leq 0\right\}=\emptyset .
$$

Therefore,

$$
\alpha^{*}(x)=\delta_{1},
$$

for each $x \in[0, K]$.

The best strategy is always to require the lowest possible intensity of payments, under the budget constraints. 


\section{Monte Carlo simulation results}

This section contains a validation of our theoretical results through simulations. Starting from empirical data, we aim at deriving the payment flow $\alpha$ which can maximize the distance to default function $V(x)$. The results will also be discussed.

The payment flow $\alpha$ is given by the product of the loan interest rate $i$ and the debt amount $D$. To fit better with the available data, we will deal with the analysis of the optimal loan interest rate $i$, which will lead to the optimal intensity of payment $\alpha$.

We here present the cases of small, medium and large companies, by considering the values $X_{0}=100, X_{0}=500$ and $X_{0}=1000$ as starting points for the dynamics of a firm's wealth.

The initial amount of the loan $D$ is assumed to be prudentially given as the $20 \%$ of the value $X(0)$. Hence we have $D=20, D=100$ and $D=200$ for small, medium and large companies, respectively.

A prudential restitution threshold $K$ can be given as the sum of the initial wealth of the firm and more than double the debt amount $D$. So we assume $K=150, K=750$ and $K=1500$ for small, medium and large companies, respectively.

Via a Monte Carlo simulation, we build 1000 different trajectories of the firm's wealth $X$ in equation (4), where we replace $\alpha$ with $i \cdot D$; to this purpose, the loan interest rate $i$ is assumed to vary in an appropriate band, accordingly with the empirical evidence, while the other parameter values are fixed:

- the lower bound $i_{L}$ of the loan interest rate should be considered null, and thus $\delta_{1}=i_{L} \cdot D=0$. We will apply $i_{L}=0$ in our simulations. In practice, the Bank of America Merrill Lynch EMU average of corporate non financial retention yield ${ }^{2}$ for firms classified as AAA is about $2.613 \%$, while for those classified as BBB is about $3.629 \%$. Therefore, we stress that a value of about $2.5 \%$ can be viewed as a lower threshold for the loan interest rate which satisfies the budget constraints in (5). Moreover, although the upper bound of the legal interest rate may be generally considered quite high, according to the country laws against usury, the loan interest rate of $3.629 \%$ is the highest used in its financing practice by Merrill Lynch. Hence, we can prudentially fix the upper

\footnotetext{
${ }^{2}$ Source: Data Stream; data refer to $9^{\text {th }}$ July 2010.
} 
bound for the loan interest rate $i_{U}$ at $5 \%$, thus $\delta_{2}=i_{U} \cdot D=1,5,10$ for small, medium and large companies, respectively;

- $\mu=1+\rho=1.001$, where $\rho$ is the revaluation rate of the company;

- $\sigma=0.01$.

The simulation procedure for the three cases of small, medium and large companies is implemented as follows:

- the Brownian Motion is discretized as $d W(t)=\Lambda * \sqrt{\Delta t}$, where $\Lambda$ is a random number extracted by a centered normal distribution and $\Delta t=1$;

- we consider a discretization of the range $[0,0.05]$ of the loan interest rate $i$ with a step equal to 0.001 ; we denote each value of $i$ as $i_{s}$ ( $s=$ $1, \ldots, 5.000)$

- we identify time-points as days and we consider 1000 points to construct each trajectory in order to analyze the evolution of the firm's wealth for a period of approximately three years;

- fixed $i_{s}, 1000$ trajectories $X_{j}^{i_{s}}(j=1, \ldots, 1000)$ are built ${ }^{3}$;

- for each $X_{j}^{i_{s}}$ we derive the time $\tau_{j}^{i_{s}}$ in which, for the first time, the trajectory of $X_{j}^{i_{s}}$ hits the barrier $\{0, K\}$.

Let $n^{i_{s}}$ be the number of the $\tau_{j}^{i_{s}}$ such that $X_{j}^{i_{s}}\left(\tau_{(0, K)}\right)=0$; we calculate for each value of $i_{s}$ the probability considered in equation (4) as follows

$$
P\left(X_{j}^{i_{s}}\left(\tau_{(0, K)}\right)=0\right)=\frac{n^{i_{s}}}{1000}=h^{i_{s}} .
$$

For each value of $i_{s}$ the average $\bar{\tau}^{i_{s}}$ of the $n^{i_{s}}$ values $\tau_{j}^{i_{s}}$ for which $X^{i_{s}}\left(\tau_{(0, K)}\right)=$ 0 is also derived.

As a result we obtain: ${ }^{4}$

\footnotetext{
${ }^{3}$ When the 1000 trajectories $X_{j}^{i_{s}}$, each made up of 1000 points, are traced, the value $i_{s}$ increases by 0.001 and then, in relation to this new value of the loan interest rate, we determine further 1000 trajectories of 1000 points, and so on.

${ }^{4}$ Data are available upon request.
} 
1. the level $i$ of the loan interest rate needed to minimize the default probability of the funded company is very close to the lower actual empirical level of the Bank of America Merrill Lynch EMU average of corporate non financial retention yield, such as to the previously quoted rate of $2.613 \%$ applied to the firms classified as AAA. In particular, we obtain that $i$ is equal to $2.694 \%, 2.591 \%$ and $2.342 \%$ for small, medium and big firms, respectively. Therefore, the optimal intensity of payment $\alpha$ is constant, as the theoretical results also show, and it is given by $\alpha^{*}=0.02694 \cdot 20=0.5388, \alpha^{*}=0.02591 \cdot 100=2.591$ and $\alpha^{*}=0.02342 \cdot 200=4.684$ for small, medium and large companies. Hence, in our case, we can also argue that the level of optimal intensity of payment $\alpha^{*}$ decreases with respect to the firm's size.

2. After performing a correlation analysis ${ }^{5}$ on the results obtained via Monte Carlo simulation for $P\left(X^{\alpha}\left(\tau_{(0, K)}\right)=0\right)$ and $\bar{\tau}^{\alpha}$ we have that:

- the probability $P\left(X^{\alpha}\left(\tau_{(0, K)}\right)=0\right)$, obviously, increases with respect to the interest rate $\alpha$;

- the average $\bar{\tau}^{\alpha}$ of the values of the $\tau_{j}^{\alpha}$ for which $X^{\alpha}\left(\tau_{(0, K)}\right)=0$, obviously, decreases with respect to $\alpha$.

\section{Discussion: the financial stability of the company}

In the analysis previously performed, no attempt has been made to describe the decision criteria adopted by the banks when granting credit to com-

\footnotetext{
${ }^{5}$ It is possible to analyze such results for each firm size in Table 1 in detail. Because of the very low level of correlation values, expected (as standard econometric theory suggests) with a remarkable amount of information as in this case, we also performed a simple linear regression analysis of $P\left(X^{\alpha}\left(\tau_{(0, K)}\right)=0\right)$ and $\bar{\tau}^{\alpha}$ on $\alpha$. In this way, the results regarding the correlation's directions have been confirmed. We report in detail them for each firm size in each of the regressions in Table 2; once more, having a considerable amount of data and because the aim of these regressions is to receive confirmation about the sign (and subsequently about the causality direction) of the regression parameters, it is possible to understand why there is a very low $R^{2}$ associated to any simple linear regression. In any case, the $t$-tests allow us to be confident of a probability of $95 \%$ in the value, and so in the sign, of any regressor's coefficient and, as a consequence, it is possible to confirm the results reported in Table 1 regarding the correlation's direction.
} 


\begin{tabular}{|c|c|c|c|}
\hline & $X_{0}=100$ & $X_{0}=500$ & $X_{0}=1000$ \\
\hline \hline$\rho\left(P\left(X^{\alpha}\left(\tau_{(0, K)}\right)=0\right), \alpha\right)$ & 0.006 & 0.015 & 0.014 \\
\hline$\rho\left(\bar{\tau}^{\alpha}, \alpha\right)$ & -0.012 & -0.0055 & -0.018 \\
\hline
\end{tabular}

Table 1: Correlations between the quantities obtained via Monte Carlo Simulation.

\begin{tabular}{|c|c|c|c|}
\hline Independent variable & $X_{0}=100$ & $X_{0}=500$ & $X_{0}=1000$ \\
\hline \hline$P\left(X^{\alpha}\left(\tau_{(0, K)}\right)=0\right)$ & $0.03(0.010)$ & $0.384(0.016)$ & $0.71(0.064)$ \\
\hline $\bar{\tau}^{\alpha}$ & $-0.00006(0.00003)$ & $-0.00014(0.000016)$ & $-0.0009(0.00006)$ \\
\hline
\end{tabular}

Table 2: Results of the Regressions between the quantities obtained via Monte Carlo Simulation. Dependent variable: $\alpha$. The Table summarizes the values of the parameters $\beta$ for the linear relation between $\alpha$ and the independent variable. Each value of $\beta$ is significative at $5 \%$ referring to a bilateral test; standard error in brackets; the number of observations is 5000 .

panies $^{6}$. In particular, we have focused on what happens after the positive decision taken by the bank to finance a firm, and the solution of the distanceto-default maximization problem does not guarantee either the achievement of the threshold $K$ (success of the firm) or the non occurrence of bankruptcy (default case). We strongly stress that, once the positive decision is taken by the bank, the analysis contained above is very general. More precisely, we have theoretically proven that the optimal strategy $\alpha^{*}(x)=\delta_{1}$ does not depend on the constitutive parameters of the wealth evolution of the firm, i.e. $x, \mu$ and $\sigma$. Needless to say, the bank checks the financial situation of the company before granting credit. This, in our opinion, is the relationship between the lender and the borrower. Aspects regarding the decision itself are out of the scope of the present study, and we leave them to future research. Indeed, a more complicated model is needed to develop this intriguing topic,

\footnotetext{
${ }^{6}$ Among the most widespread techniques using for assessing the credit risk, refer to the multivariate econometric and statistical methods, such as logit and probit regressions, linear and quadratic discriminant analysis and cluster analysis; reviews on potentialities, limits and applications of such procedures in relation to the credit scoring are available in Altman (1968, 1989), Altman and Saunders (1998), Altman and Sabato (2005), Altman et al. (1977, 1998), Carrara and Cavalli (1996), Gilbert et al. (1990) and Gordy (2000, 2003). Recent studies have considered some new non-parametric methods such as neural networks, support vector machines, classification trees and mathematical programming; among the others, Baesens et al. (2003) and Baourakis et al. (2009).
} 
and the complex nature of this economic problem may be captured only by inserting more ingredients into the analysis. However, we can also argue that the riskier the company, the more dangerous it is for the bank to grant credit.

For the analysis of the implications of the financial instability of the funded company on the solidity of the financing bank, we first need to point out the conditions related to a firm's financial stability. In our simplified model, three factors may affect the health of a company:

- the parameter $\mu$ synthesizes the deterministic trend associated to the evolution of the firm's wealth. The larger the value of $\mu$, the stronger the positive trend. Therefore, a large value of $\mu$ is a desirable condition to contribute to a safe company;

- the initial value $x$ can be interpreted as a measure of the initial distanceto-default of the company. As $x$ approaches 0 , then it is more difficult for the firm to reach the upper bound $K$ and restitute the debt. Hence, we can say that a great initial firm value may be viewed as a condition for the financial stability of the funded company;

- the volatility $\sigma$ is a measure of the financial health of the company, in that the higher the level of volatility, the riskier the dynamics of the firm's wealth.

For the sake of simplicity and without loss of generality, we discuss how the financial distress of the funded firm influences the value function by allowing just one of the parameters to vary, leaving the others unchanged.

We have already theoretically obtained that the value function is increasing with respect to $x$ (see Remark 7). To discuss the behavior of $V$ with respect to $\mu$ and $\sigma$, we proceed by numerical approximation, as the integrals appearing in (13) involve complicated truncated Gamma functions.

Now, fix $x \in[0, K]$. According to (13) and (23), define:

$$
\tilde{V}_{x}:[0,+\infty) \times[0,+\infty) \rightarrow[0,1] \text { such that }(\sigma, \mu) \mapsto \tilde{V}_{x}(\sigma, \mu),
$$

with

$$
\tilde{V}_{x}(\sigma, \mu)=H \cdot \frac{\int_{0}^{x} \exp \left\{-\frac{2 \delta_{1}}{\sigma^{2} t}-\frac{2 \mu}{\sigma^{2}} \log t\right\} \mathrm{d} t}{\int_{0}^{K} \exp \left\{-\frac{2 \delta_{1}}{\sigma^{2} t}-\frac{2 \mu}{\sigma^{2}} \log t\right\} \mathrm{d} t} .
$$




\begin{tabular}{|c|c|c|c|c|}
\hline$K$ & $x$ & $\delta_{1}$ & $\bar{\mu}$ & $\bar{\sigma}$ \\
\hline \hline 100 & 50 & 0.25 & 1.001 & 0.04 \\
\hline
\end{tabular}

Table 3: Parameter set.

We consider two values for $\mu$ and $\sigma$, say $\bar{\mu}$ and $\bar{\sigma}$, and study the behavior of $\tilde{V}_{x}(\bar{\sigma}, \mu)$ and $\tilde{V}_{x}(\sigma, \bar{\mu})$. In the former case, $\mu \in[0.0001,0.001]$, while in the latter case $\sigma \in[0.1,10]$.

Table 3 contains the parameter set used to perform our simulations. Figure 1 shows the shape of $V_{x}(\bar{\sigma}, \mu)$, while Figure 2 contains the graph of $V_{x}(\sigma, \bar{\mu})$.

\section{INSERT FIGURE 1 ABOUT HERE.}

\section{INSERT FIGURE 2 ABOUT HERE.}

The scenario analysis of the firm's default probability is particularly useful if the dynamics of the firm's wealth is partially unclear and the parameters $\mu$ and $\sigma$ are unknown. The results agree in stating that the value function decreases as the company becomes less safe. This finding can be interpreted as a recommendation for the financing bank. Indeed, if the preliminary check of financial stability of the company performed by the bank evidences that the adoption of the best strategy leads to a small probability of company default, then the funded firm may be in a situation of severe financial distress. This result sounds reasonable, in that one can conjecture that a large distance-todefault at the optimal level of intensity of payments is a companion element of a high default probability when the intensity of payment is not optimal. Substantially, the lower the minimum default probability, the higher the maximum default probability, where minimum and maximum have to be intended with respect to $\alpha$. Therefore, a low level of value function may be associated with a unstable firm, with wealth which is particularly sensitive to the variations of economic parameters.

Further studies need to be carried out in order to elaborate a definitive theory on this topic.

\section{Conclusions}

In this work, the best intensity of payment which a bank has to apply to a funded company has been explored. For this reason, a dynamic stochastic 
optimization problem has been constructed and developed. The aim of the legal financier is to maximize the distance-to-default function, i.e. the probability that the funded firm repays its debt in full. The solution strategy is analytically attained: the best intensity of payment is the lowest possible which satisfies the bank's budget constraints.

The theoretical results are confirmed when the optimization problem is tackled via a Monte Carlo simulation procedure involving empirical data. In this case, findings show that (i) the optimal level of the payment flow is very close to the one generated by lower bound of the actual interest rate variation range, and it decreases as the firm's size increases; (ii) the default probability of the company increases with respect to the level of payment flow; (iii) the average of the times values for which the company fails decreases as the intensity of payment increases.

\section{References}

[1] Altman EI. Financial Ratios, Discriminant Analysis and the Prediction of Corporate Bankruptcy. Journal of Finance 1968; 68; no. 4; 589-611.

[2] Altman EI. Measuring Corporate Bond Mortality and Performance. Journal of Finance 1989; 44; no. 4; 909-922.

[3] Altman EI, Caouette JB, Narayan P. Managing Credit Risk. The Next Great Financial Challenge. Wiley Frontiers in Finance; 1998.

[4] Altman EI, Haldeman RG, Narayan P. ZETA Analysis: A New Model to Identify Bankruptcy Risk of Corporations. Journal of Banking and Finance 1977; 1; 29-54.

[5] Altman EI, Sabato G. Effects of the New Basel Capital Accord on Bank Capital Requirements for SMEs. Journal of Financial Services Research $2005 ; 28 ; 15-42$.

[6] Altman EI, Saunders A. Credit Risk Measurement: Developments over the Last 20 Years. Journal of Banking and Finance 1998; 21; 1721-1742.

[7] Anderson R, Sundaresan S. A comparative study of structural models of corporate bond yields: an exploratory investigation. Journal of Banking and Finance 2000; 24; 255-269. 
[8] Baesens B, Gestel TV, Viaene S, Stepanova M, Suykens J, Vanthienen J. Benchmarking state-of-the art classification algorithms for credit scoring. Journal of Operational Research Society 2003; 54; 627-635.

[9] Baourakis G, Conisescu M, Van Dijk G, Pardalos PM, Zopounidis C. A multicriteria Approach for Rating the Credit Risk of Financial Institutions. Computational Management Science 2009; 6; 347-356.

[10] Barone R, Cerqueti R, Quaranta AG. Illegal financier and usurer behavior. European Journal of Law and Economics (in press).

[11] Borkar VS. Optimal control of diffusion processes. Pitman Research Notes in Mathematics Series, Longman Scientific \& Technical: Harlow; 1989.

[12] Brennan M, Schwartz E. Corporate income taxes, valuation, and the problem of optimal capital structure. Journal of Business 1978; 51; 103114.

[13] Caballero RJ, Pindyck RS. Uncertainty, Investment, and Industry Evolution. International Economic Review 1996; 37; 641-662.

[14] Carrara D, Cavalli E. Bankruptcy Prediction. In: Modelling Techniques for Financial Markets and Bank Management. Eds. M. Bertocchi, E. Cavalli and S. Komlosi. Springer, Heidelberg; 1996.

[15] Cifarelli MD, Masciandaro D, Peccati L, Salsa S, Tagliani A. Success or failure of a firm under different financing policies: a dynamic stochastic model. European Journal of Operational Research 2002; 136; no. 3; 471482.

[16] Dixit A. Hysteresis, Import Penetration, and Exchange-Rate PassThrough. Quarterly Journal of Economics 1989; 104, 205-228.

[17] Duan JC. Maximum Likelihood Estimation using Price Data of the Derivative Contracts. Mathematical Finance 1994; 4, 155-167.

[18] Duan JC, Gautier G, Simonato S, Zaanouns S. Estimating Merton's Model by Maximum Likelihood with Survivorship Consideration. European Financial Association Conference - Maastricht Meeting Papers no. 4190, 2004. 
[19] Fleming WH, Soner HM. Controlled Markov processes and viscosity solutions. Applications of Mathematics (New York), Springer-Verlag: New York; 1993.

[20] Gilbert L, Menon K, Schwartz K. Predicting Bankruptcy for Firm in Financial Distress. Journal of Business Finance and Accounting 1990; Spring.

[21] Gordy MB. A Comparative Anatomy of Credit Risk Models. Journal of Banking and Finance 2000; 24; no. 1-2; 119-149.

[22] Gordy MB. A Risk-factor Model Foundation for Ratings-Based Bank Capital Ratios. Journal of Financial Intermediation 2003; 12; 199-232.

[23] Krouse CG, Lee WY. Optimal Equity Financing of the Corporation. Journal of Financial and Quantitative Analysis 1973; 8; 539-563.

[24] Krylov NV. Controlled diffusion processes. Springer-Verlag: New York; 1980.

[25] Leahy J. The Optimality of Myopic Behavior in a Competitive Model of Entry and Exit. Discussion Paper No. 1566, Harvard University, August 1991.

[26] Leland H. Corporate debt value, bond covenants, and optimal capital structure. Journal of Finance 1994; 49; no. 4; 1213-1252.

[27] Leland H, Toft KB. Optimal capital structure, endogenous bankruptcy, and the term structure of credit spreads. Journal of Finance 1996; 51; no. 3; 987-1019.

[28] Li Y, MacLean LC, Ziemba WT. Security and wealth aspects of optimal capital growth models with minimum expected time criteria. Mimeo, University of British Columbia, 1996.

[29] Longstaff F, Schwartz E. A simple approach to valuing risky debt. Journal of Finance 1995; 50; 789-821.

[30] Masciandaro, D. In Offense of Usury Laws: Microfoundations of Illegal Credit Contracts. European Journal of Law and Economics 2001; 12; no. $3 ; 193-215$. 
[31] Masciandaro D, Peccati L, Tagliani A. Why usury can be cheaper? Atti del XXI Convegno A.M.A.S.E.S, Rome, Italy; 1997; 453-468.

[32] Modigliani F, Miller M. The cost of capital corporation finance, and the theory of investment. American Economic Review 1958; 48; 261-277.

[33] Merton RC. On the pricing of corporate debt: the risk structure of interest rates. Journal of Finance 1974; 29; 449-470.

[34] Øksendal, B., Stochastic differential equations. An introduction with applications, Fourth edition, Universitext, Springer-Verlag, Berlin; 1995.

[35] Pardalos PM, Tsitsiringos V. (Eds.) Financial Engineering, E-commerce and Supply Chain (Applied Optimization). Kluwer Academic Publishers; 2002.

[36] Sethi SP. Optimal Equity Financing Model of Krouse and Lee: Corrections and Extensions. Journal of Financial and Quantitative Analysis 1978; 13; no. 3 ; 487-505.

[37] Sethi SP, Taksar MI. Optimal Financing Of A Corporation Subject To Random Returns. Mathematical Finance 2002; 12; no. 2; 155-172.

[38] Yong J, Zhou XY. Stochastic controls. Hamiltonian systems and HJB equations. Applications of Mathematics, Springer-Verlag: New York; 1999. 